\title{
Metallographische Mitteilungen aus dem Institut für physikalische Chemie der Universität Göttingen.
}

\author{
LXII. \\ Über Vanadin-Eisenlegierungen. \\ Von \\ R. Vogel und G. Tammann. \\ Mit 2 Figuren im Text.
}

Zur Aufklärung der Konstitution der Vanadin-Eisenlegierungen wurde ihr Zustandsdiagramm ausgearbeitet und ihre Struktur mikroskopisch untersucht. Als Ausgangsmaterial für die Darstellung der $\mathrm{Fe}$-reicheren Legierungen diente ein aluminothermisch dargestelltes Ferrovanadin, das einer Analyse des Herrn Dr. LAdTSCH zufolge $72.9 \%$ Fe und $26.8 \% \mathrm{~V}$ enthielt. Auf der Abkühlungskurve dieser Legierung fand sich zwischen 1451 und $1441^{\circ}$ ein Krystallisationsintervall, die Legierung ist demnach als Glied einer Reihe von Mischkrystallen aufzufassen, was durch die Struktur der Legierung, welche nur eine einzige Krystallart enthielt, bestätigt wird. Mit Hilfe dieser Legierung wurden die Fe-reicheren Legierungen durch Zusammenschmelzen mit Eisen in Porzellanröhren dargestellt und ihre Abkühlungskurven bestimmt. Die Darstellung der Legierungen mit höherem Vanadingehalt versuchten wir zunächst durch Zusammenschmelzen von Eisen und Vanadin. Als Ausgangsmaterial wurde Eisen verwandt, dessen Zusammensetzung früher ${ }^{1}$ angegeben ist. Die Darstellung des Vanadins geschah auf aluminothermischem Wege. Vanadinsaures Ammonium ron KaHLBaUm wurde in einer Porzellanschale so lange auf etwa $250^{\circ}$ erhitzt, bis aus dem gelb. braunen Produkt alles Ammoniak entwichen war; hierzu war etwa 10stündiges Erhitzen erforderlich. Erböhte man die Temperatur noch weiter, so schmolz das sich bildende $\mathrm{V}_{2} \mathrm{O}_{5}$ und war dann schwierig von der Porzellanschale abzulösen. Beim Arbeiten mit

'Z. anorg. Chem. 55 (1907), 387. 
$\mathrm{V}_{\mathrm{g}} \mathrm{O}_{5}$ ist Vorsicht zu empfehlen, da es scheint, als ob der eingeatmete $\mathrm{V}_{2} \mathrm{O}_{5}$-Staub Entzündung der Schleimhäute hervorruft. Zur aluminothermischen Reduktion wurden auf $50 \mathrm{~g}$ Metall berechnete Mengen von $\mathrm{V}_{2} \mathrm{O}_{5}$ und Aluminiumgries $(5 \% \mathrm{Al}$ weniger als theoretisch erforderlich, um die Bildung einer Legiermng von $\mathrm{V}$ und Al zu verhindern) innig gemengt und in hessischen Tiegeln abgebrannt. Die Reaktion verlief glatt innerhalb 2-3 Sekunden. Das Metall flofs dabei leicht zu einem einzigen Regulus zusammen, dessen Gewicht $60-75 \%$ der möglichen Ausbeute betrug.

\section{Eisen-Vanadin-Siliciumlegierungen.}

Versuche, Legierungen mit mehr als etwa $30 \% \mathrm{~V}$ durch $\mathrm{Zu}$ sammenschmelzen von Eisen oder der $26.8 \%$ igen Legierung mit Vanadin darzustellen, führten zu keinem Resultat, denn das Vanadin bedeckte sich leicht, auch im H-Strom, mit einer schwarzen Haut. Das Eisen schmolz leicht zusammen und löste nur wenig Vanadin, auch wenn man die Temperatur bis über den Schmelzpunkt des Vanadins auf $1800-1900^{\circ}$ steigerte. Infolgedessen wurden die Legierungen mit mehr als $30 \% \mathrm{~V}$ durch aluminothermische Reduktion berechneter Gemische von $\mathrm{Fe}_{2} \mathrm{O}_{3}$ und $\mathrm{V}_{2} \mathrm{O}_{5}$ dargestellt.

Die Reaktion verlief auch hier stets glatt, ihre Geschwindigkeit verringerte sich mit abnehmendem Vanadingehalt der Legierungen und die Ausbeute hielt sich regelmäfsig in den Grenzen von $60-76 \%$. Die in ungeschützten hessischen Tiegeln hergestellten Legierungen sowie auch das Vanadin enthielten zwischen 6.03 und $8.93 \%$ Silicium, welches während der Reduktion aus den Tiegelwandungen aufgenommen wurde. Aufserdem stellte sich heraus, dafs sich das berechnete Verhältnis von Eisen und Vanadin bei der Reaktion um durchschnittlich $8.5 \%$ zugunsten des Eisens verschob. Die Legierungen bis zu $50 \% \mathrm{~V}$ wurden in Porzellanröhren, die vanadinreicheren in Magnesiaröhren umgeschmolzen, und da die Schmelzen dünnfiüssig waren und das Porzellanschutzrohr des Thermoelementes wenig angriffen, so gelang es ohne besondere Schwierigkeiten ihre Abkühlungskurven zu bestimmen. Über $70 \% \mathrm{~V}$ hinaus, wo die Temperatur von $1600^{\circ}$ überschritten werden mulste und das Thermoelement infolge Weichwerdens des Porzellanschutzrohres nicht mehr zu brauchen war, wurde versucht, die Abkiihlungskurven photometrisch mit Hilfe des Wanner-Pyrometers zu bestimmen. Bei einiger Übung gelingt es, ziemlich regelmälsig verlaufende Abkühlungskurven zu 
erhalten, auf denen die durch Krystallisation verursachten Wïrmeeffekte deutlich wahrnehmbar waren, doch kommen natürlich auch grolse Einstellungsfehler bis $40^{\circ}$ nicht selten vor. Die Bedingung, unter welcher die Angaben des Pyrometers allein streng richtig sind, dals die zu untersuchende Strahlung die eines absolut schwarzen Körpers sei, ist hier nicht ganz erfüllt. Daher sind die wahren Temperaturen wohl noch etwas höher gewesen als die gemessenen. Dämpfe, welche die von der Oberfläche der Schmelze ausgesandte Strahlung hätten absorbieren können, wurden nicht wahrgenommen.

Die Legierungen mit $0-50 \% \mathrm{~V}$ wurden auf $1600^{\circ}$, die mit $50-100 \% \mathrm{~V}$ auf $1600-1900^{\circ}$ erhitzt und die Abkühlungskurven von $1600^{\circ} \mathrm{ab}$ ab gewöhnlich bis etwa $1100^{\circ}$ verfolgt. Alle Temperaturen sind auf die Skala des Lufthermometers reduziert. Die Resultate sind in Tabelle 1 wiedergegeben und in Fig. 1 graphisch

Tabelle 1.

\begin{tabular}{|c|c|c|c|c|c|}
\hline \multicolumn{3}{|c|}{$\begin{array}{l}\text { Gehalt der Legierungen } \\
\text { in Gewichtsprozenten }\end{array}$} & \multicolumn{2}{|c|}{$\begin{array}{c}\text { der Krystallisation } \\
\text { in }^{*}\end{array}$} & $\begin{array}{l}\text { Unwand } \\
\text { lung }\end{array}$ \\
\hline$* 24.51$ & 67.99 & $7 . \overline{2}$ & 1893 & 1365 & 1120 \\
\hline 29.13 & 63.37 & 7.5 & 1357 & 1335 & 1170 \\
\hline *33.76 & 58.74 & 7.5 & 1339 & - & \\
\hline 38.38 & 54.12 & 7.5 & $13 \overline{0} 0$ & 1335 & \\
\hline 43.01 & 49.49 & 7.5 & 1367 & 1843 & 1170 \\
\hline 47.63 & 44.87 & 7.5 & $137 \pi$ & 1351 & 1220 \\
\hline${ }^{*} 52.26$ & 40.24 & 7.5 & 1379 & $\begin{array}{c}1371 \\
\text { Haltepunkte: }\end{array}$ & \\
\hline 56.88 & 35.62 & 7.5 & 1451 & 1365 & \\
\hline 61.51 & 30.99 & 7.5 & 1524 & 1398 & \\
\hline 80.01 & 12.49 & 7.5 & 1630 & 1340 & \\
\hline$* 92.50$ & - & 7.5 & 1680 & - & \\
\hline
\end{tabular}

* Analysiert.

dargestellt. Da die Vanadin-Eisenlegierungen, auf die sich Fig. 1 hezieht, durchweg $6-8 \%$ Silicium enthielten, so ist auch für die Konzentration $\mathrm{V}=0$ nicht der Schmelzpunkt des reinen Eisens, sondern der eines Silicium-Eisens mit $7.5 \%$ Silicium eingetragen; auf den fast konstanten Si-Gehalt ist auf der Konzentrationsachse keine Rücksicht genommen. Fig. 1 bezieht sich also auf eine Legierungsreihe von nahezu konstantem Si-Gehalt, oder wir haben es hier mit einem Schnitt durch das Dreistoffsystem Eisen-VanadinSilicium parallel der Eisen-Vanadinachse zu tun. 
Las Verständnis der Vorgänge in diesem Schnitt wird wesentlich erleichtert, wenn wir das Resultat der Untersuchung der Si freien Eisen-Vanadinmischungen vorwegnehmen. Vanadin und Eisen bilden eine lückenlose Reihe von Mischkrystallen und die Kurve des Beginnes der Krystallisation dieser Reihe von Mischkrystallen hat ein Minimum bei $32 \%$ V. Durch Beimengung von Silicium wird dieses Minimum, wie man beim Vergleich von

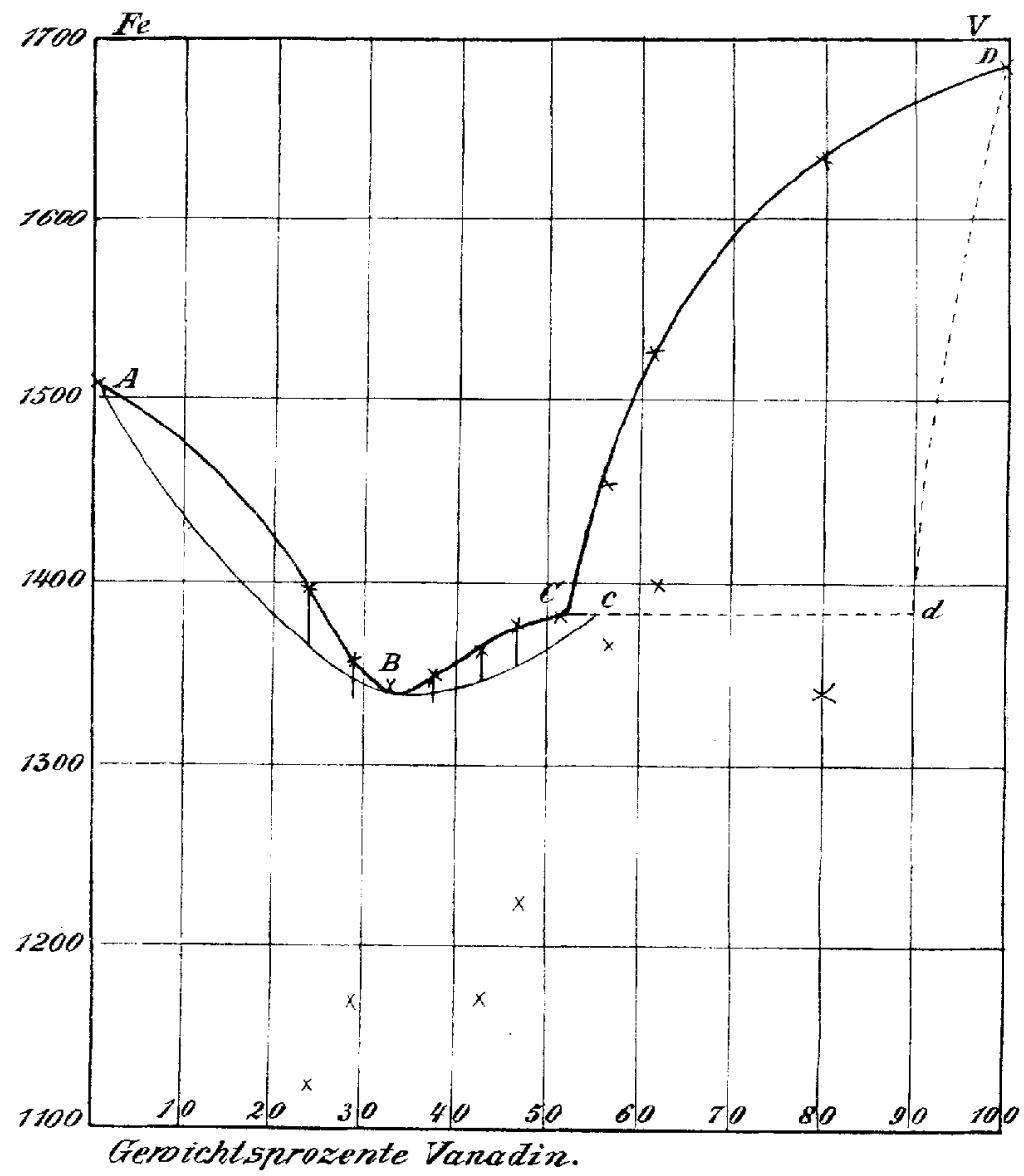

Fig. 1. Schmelzpunkte der Vanadin-Eisenlegierungen mit 7.5\% Silicium.

Fig. 1 und 2 sieht, zu tieferen Temperaturen verschoben. Aufserdem tritt bei $1380^{\circ}$ eine Mischungslücke auf, welche von etwa $55 \%$ bis vielleicht $90 \%, \nabla$ reicht. Für diese Auffassung sprechen folgende Gründe: 
1. Die von $1300^{\circ}$ abgeschreckten Legierungen mit $20-50 \% \mathrm{~V}$ (V-ärmere Legierungen mit einem Si-Gehalt von $7.5 \%$ wurden nicht untersucht) bestehen aus unter sich homogenen Polyedern. ${ }^{1}$ Es

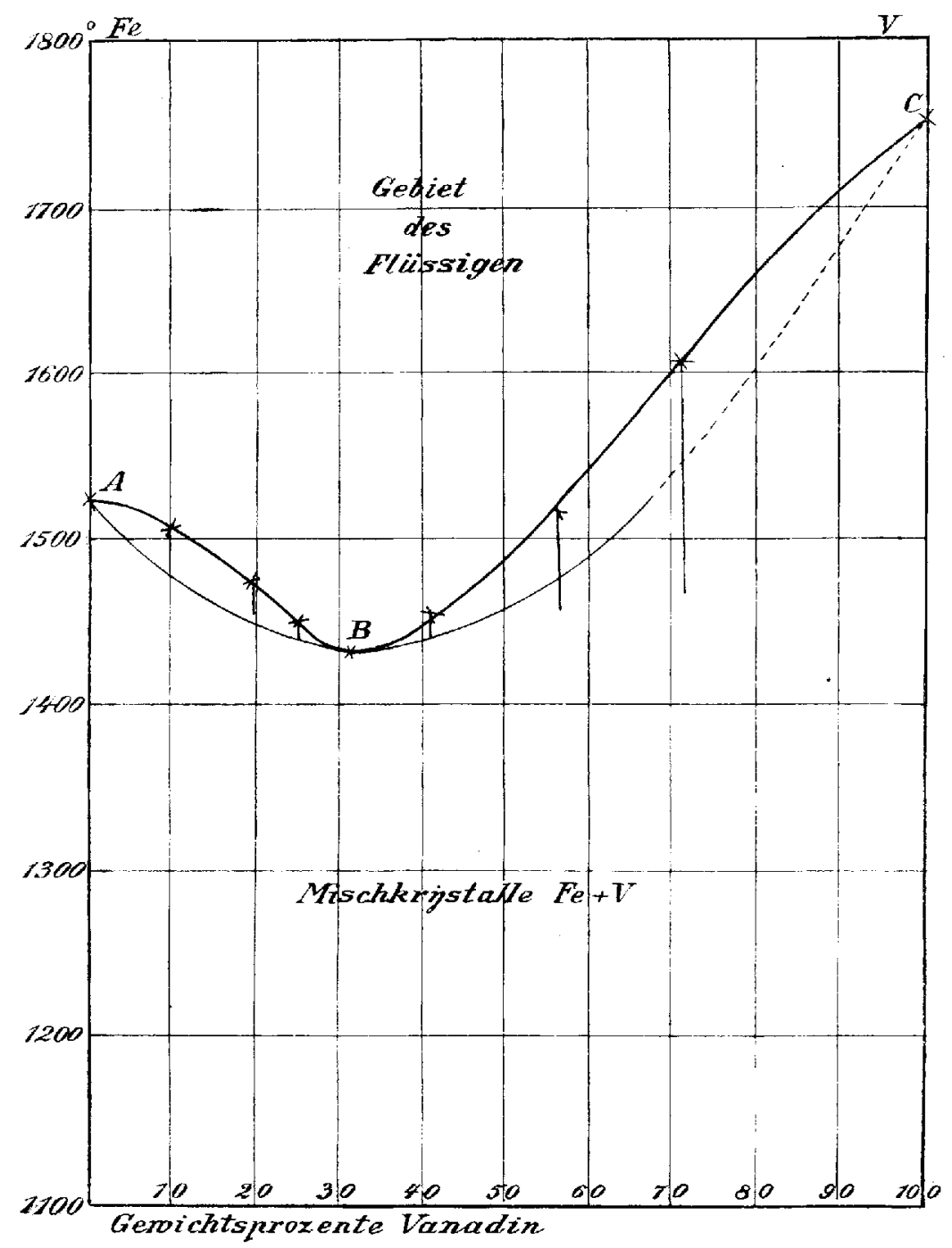

Fig. 2. Zustandsdiagramm der Vanadin-Eisenlegierungen.

1 Zur mikroskopischen Untersuchung geeignete Schliffe der Fe-V-SiLegierungen wurden erhalten durch Schleifen der Legierungen auf Glas unter Verwendung von Seifenlösung und feinstem Schmirgel. Als Ätzmittel diente erhitztes Königswasser. 
liegt also in diesem Konzentrationsgebiet eine kontinuierliche Reihe von Mischkrystallen vor, worauf ja auch die thermischen Erscheinungen bei der Krystallisation der Schmelzen hindeuten.

2. beobachtet man in dem Gebiet von $55-80 \% \mathrm{~V}$ zwei deutliche Wärmeeffekte auf den Abkühlungskurven. Der eine entspricht dem Beginn der Krystallisation auf der Kurve $C D$ und der andere tritt ziemlich unregelmälsig etwa bei der Temperatur der Horizontalen $C d$ ein. In diesem Gebiet enthalten die Legierungen auch nach dem Abschrecken zwei verschiedene Strukturelemente, welche wir wohl als die gesättigten Mischkrystalle $e$ und $d$ ansprechen können.

In den krystallisierten Legierungen mit $20-52 \% \mathrm{~V}$ tritt zwischen 1100 und $1250^{\circ}$ eine Umwandlung auf. Kühlt man diese Legierungen langsam $a b$, so werden sie mit Ausnahme der Legierung mit dem minimalen Schmelzpunkt $B$, welche $32 \% \mathrm{~V}$ enthält, inhomogen. Auf den Abkühlungskurven konnte aber ein thermischer Effekt, der dieser Änderung der Struktur entspricht, nicht aufgefunden werden. Deshalb wurde die Temperatur der Strukturänderung für mehrere Legierungen durch Abschrecken von verschiedenen Temperaturen untersucht. Die auf thermischem Wege nicht auffindbare Temperatur der Umwandlung wurde in dieser Weise für vier Legierungen festgestellt. Ihre Temperaturen sind in Fig. 1 durch die Kreuze zwischen 1100 und $1250^{\circ}$ bezeichnet. Über die Ursachen dieser Umwandlung könnten auf Grund unserer Beobachtungen verschiedene Vermutungen geäufsert werden. Eigentümlich ist es, dals die Legierung mit dem minimalen Schmelzpunkt sowohl nach schneller als auch nach langsamer Abkühlung homogen bleibt, während sich in den Legierungen mit mehr oder weniger Vanadin bei langsamer Abkühlung eine Strukturänderung vollzieht. Das eine Ende der Mischungslücke haben wir in Fig. 1 zu etwa $55 \% \mathrm{~V}$ angenommen, weil bei dieser Konzentration die Kurve des Endes der Krystallisation die Horizontale $C d$ schneidet. Für die Lage des anderen Findes der Mischungslücke $d$ haben wir keinerlei Anhaltspunkte. Doch dafs auch siliciumhaltiges Vanadin im krystallisierten Zustande Eisen aufzunehmen rermag, ist ja sehr wahrscheinlich.

Schliel'slich sei bemerkt, dafs die aluminothermisch dargestellten Si-haltigen Legierungen beim Umschmelzen ihre Struktur nicht veränderten und sich auch betreffs der Umwandlung zwischen 1100 and $1250^{\circ}$ genau so verhalten wie die Legierungen nach dem Umschmelzen. 


\section{Fisen-Vanadinlegiernngen.}

Nahezu siliciumfreie Legierungen erhielten wir auf aluminothermischem Wege, wenn der Tiegel, in welchem die Reaktion stattfand, mit einer Futterung aus "geschmolzener" Magnesia (von der Deutschen Gold- und Silberscheideanstalt) versehen wurde. Die Magnesia wurde mit Wasser zu einem Brei angerührt und die Fiutterung langsam an der Luft trocknen gelassen, da sie bei raschem Trocknen leicht rissig wird.

Zufolge Analyse betrug der Siliciumgehalt der Legierungen jetzt nur noch durchschnittlich $1 \%$. Vermutlich rührt der Si-Gehalt von Verunreinigungen der zur Futterung der Tiegel verwandten Magnesia her, in welcher nicht unerbebliche Mengen ron Kieselsäure und Schwefel vorhanden waren. Daher diurfte die Magnesia usta, obwohl die mit ihr hergestellte Fütterung leicht abbröckelt und rissig wird, wegen ihrer grölseren Reinheit vorzuziehen sein. Das Umschmelzen und die Aufnahme der Abkühlungskurven war bei diesen Legierungen wesentlich schwieriger als bei den stark Si-haltigen Legierungen, da jene Schmelzen weniger dìnnflüssig waren und die einzelnen Metallstücke schwer zu einem einheitlichen Regulus zusammentiossen.

Die Resultate der thermischen Untersuchung sind aus Tabelle 2 und Fig. 2 ersichtlich.

Tabelle 2.

\begin{tabular}{c|c|c}
\hline \hline $\begin{array}{c}\text { V-Gehalt der Legierungen } \\
\text { in Gewichtsprozente }\end{array}$ & $\begin{array}{c}\text { Beginn } \\
\text { der Krystallisation in }\end{array}$ \\
\hline \hline 0 & \multicolumn{2}{|c}{$\begin{array}{c}\text { Ende } \\
\text { Smp. von Eisen } 1525\end{array}$} \\
10 & 1509 & 1479 \\
20 & 1475 & 1458 \\
26.8 & 1451 & 1441 \\
$* 31.5$ & 1435 & - \\
41.5 & 1458 & 1444 \\
$* 51.5$ & 1503 & 1460 \\
56.5 & 1517 & 1465 \\
$* 71.5$ & 1608 & $?$ \\
$* 100$ & Smp. von Vanadin 1750
\end{tabular}

Die Kurve des Beginnes der Krystallisation $A B C$ hat ein ausgeprägtes Minimum. Von dem Minimum $B$ steigt die Schmelzkurve ziemlich geradlinig zum reinen Vanadin. Der Schmelzpunkt des Vanadins, welcher gewöbnlich zu $1680^{\circ}$ angenommen wird, wurde 
durch Aufnahme der Abkuhhlungskurve des geschmolzenen Vanadins auf photometrischem Wege bestimmt. Hierzu wurde die Temperatur anf $1950^{\circ}$ gesteigert; wiederholte Versuche ergaben regelmälsig einen deutlichen Helligkeitshaltepnnkt von 50 Sek. Zeitdauer zwischen 1700 und $1800^{\circ}$. Das Mittel aus den verschiedenen Beobachtungen ergibt $1750 \pm 30^{\circ}$. Da auch dieses Vanadin zufolge der Analyse $1.21 \% \mathrm{Si}$ enthielt, so ist dieser Wert für den Schmelzpunkt von Vanadin wahrscheinlich etwas zu niedrig. Vanadin schmilzt also höher als bisher angenommen wurde. Vermutlich enthielt das bei den fruheren Schmelzpunktsbestimmnngen verwandte Vanadin grölsere Mengen Silicium oder andere Beimengungen. Aufserdem trat auf den Abkthlungskurven des Vanadins, und 2war nur auf denen des Vanadins bei etwa $1650^{\circ}$ ein zweiter Haltepunkt auf, dessen Zeitdauer bei wiederholtem Umschmelzen desselben Regulus sehr deutlich zunahm. Die Ursache dieses Haltepunktes dürfte daher wohl durch Lossung der Substanz des Magnesiaschmelzrohres oder durch die Aufnahme von Oxyden oder Nitriden bedingt sein. Für diese Annahme spricht auch die Veränderung, welche die Struktur des Vanadins bei wiederholtem Erhitzen über die Schmelztemperatur erleidet. Wahrend man vor dem Umschmelzen anf der geătzten Schliffläche des Vanadins nur grolse Polygone, getrennt durch feine dunkle Linien erblickt, gewahrt man nach dem Schmelzen, besonders nach den Rändern des Schliffes zu ein zweites Strukturelement, das sich beim Atzen mit $\mathrm{HNO}_{3}$ schwarz färbt.

Alle Vanadinstahle krystallisieren ihren Abkühlungskurven zufolge mit Ausnahme der niedrigst schmelzenden Legierung mit $32 \% \mathrm{~V}$, welche bei einer Temperatur vollständig krystallisiert, in Temperaturintervallen und bestehen demnach aus einer ununterbrochenen Reihe von Mischkrystallen. Eisen und Vanadin sind also sowohl in flissigem als auch in festem Zustande in allen Verhältnissen mischbar.

Durch die mikroskopische Untersuchung der Struktur ${ }^{1}$ der Vanadinstahle wird dieser Befund im allgemeinen die aus der $26.8 \% \mathrm{~V}$ enthaltenden Legierung hergestellten $\nabla$-ärmeren Legierungen bestanden durchweg aus unter sich fast homogenen Polyedern. Anch dle aluminnthermisch hergestellten Legierungen mit mehr als $30 \% \nabla$ hatten diese Struktur, nur ausnahmsweise zeigten sich

1 Die Herstellung der Schliffe bereitet im Gegensatz zu den zuerst dargestellten stark Bi-haltigen Legierungen keinerlei Schwierigkeiten. Die Schliffflächen liefsen sich leicht mit $\mathrm{HNO}_{3}$ ätzen. 
zwischen den Trennungslinien der Polygone verschwindende fremde Beimengungen. Nach dem Umschmelzen der aluminothermisch hergestellten Legierungen im Magnesiarohr trat hauptsächlich an der Rinde der Legiernngen zwischen grolsen homogenen Krystallen ein Strukturelement ron eutektischen Gefüge auf, welches sich wahrscheinlich entweder durch Aufnahme von Kohle oder von Oxyden gebilden hatte.

Die Vanadinstahle weisen glänzende grobkrystallinische Bruchflachen auf und sind mit Ausnahme der sehr Fe-reichen hart und ziemlich spröde, lassen sich jedoch noch feilen und etwas hämmern ohne zu zerspringen. Ein Siliciumgehalt von $7.5 \%$ steigert die Hhirte und Sprödigkeit soweit, dafs sie sich nicht mehr bearbeiten lassen und beim Hämmern wie Glas zerspringen. Gleichzeitig erhöht sich ihre Widerstandsfähigkeit gegen Săuren. Die nahezu Si-freien Legierungen werden ron $\mathrm{HNO}_{3}$ leicht angegriffen, die mit $7.5 \% \mathrm{Si}$ nur von erhitztem Königswasser. Die Umwandlungen des reinen Eisens sind schon bei einer Legierung mit $10 \% \mathrm{~V}$, deren $\mathrm{Ab}$ kühlungskurve bis $500^{\circ}$ verfolgt wurde, thermisch nicht mehr nachweisbar.

Dals Eisen und Vanadin eine lückenlose Reihe von Misch. krystallen bilden, wird gewils jedem auffallen, der an die MENDELEJEFFsche oder Lothab MeYersche Anordnung der Elemente denkt. Diese Anordnungen bringen aber die Elemente verschiedener natürlicher Gruppen im engeren Sinn durcheinander. Orientiert man sich über die Stellung ron Eisen und Vanadin in einer Anordnung, welche besondere Rucksicht auf die natürlichen Gruppen im engeren Sinne nimmt, indem sie wie die STaIGmüLLER sche Anordnung nur die Elemente je einer natürlichen Gruppe zusummenstellt, so kommen die natürlichen Gruppen im engeren Sinn der Elemente mit hohem Schmelzpunkt in die Mitte des periodischen Systemes zu steben, wie die folgende Anordnung zeigt.

$\begin{array}{lcllllll}\mathrm{Si} & & & & & & & \\ \mathrm{Ti} & \mathrm{V} & \mathrm{Cr} & \mathrm{Mn} & \mathrm{Fe} & \mathrm{Co} & \mathrm{Ni} & \mathrm{Cu} \\ \mathrm{Zr} & \mathrm{Nb} & \mathrm{Mo} & & \mathrm{Ru} & \mathrm{Rh} & \mathrm{Pd} & \mathrm{Ag} \\ & \mathrm{Ta} & \mathrm{W} & & \mathrm{Os} & \mathrm{Ir} & \mathrm{Pt} & \mathrm{Au}\end{array}$

Wir wissen, dafs Eisen mit Kobalt und Nickel, mit Mangan und Chrom" lückenlose Reiben ron Mischkrystallen bilden; dafe das

1 Die aluminothermisch dargestellten Legierungen, Trextgouke und Tamraxs, Z. anorg. Chem. 55 (1907), 402.

Z. anory. Chom. Bd. 68 . 
dem Cr benachbarte Vanadin ebenfalls mit Eisen eine lückenlose Reihe von Mischkrystallen bildet, ist hiernach nicht mehr auffallend, da ja sowohl Titan als auch Silicium, welche dem Eisen noch ferner stehen, mit krystallisiertem Eisen, wenn auch nicht in allen Verhältnissen so doch begrenzte Reihen von Mischkrystallen bilden. Die Tatsache, dafs die Elemente rom Titan (exkl.) bis zum Kupfer (exkl.) in obiger Anordnung mit dem Eisen lückenlose Reihen von Mischkrystallen bilden, hat ihren Grund wohl nicht darin, dafs diese Elemente einander chemisch besonders nahe stehend, also gleich konstituiert sind, sondern ist wohl auf die hohe Schmelztemperatur dieser Elemente zurlickzufuhren. ${ }^{1}$

' Z. anorg. Ohem. 53 (1907), 446.

Göttingen, Institut fïr physikalische Ohemie.

Bai der Redaktion eingegengen am 2. Mărz 1908. 